\title{
2 Chronic social stress leads to altered sleep homeostasis in
}

\section{3 mice}

$4 \quad$ Nadja Olini ${ }^{1,2}$, Iru Rothfuchs ${ }^{1}$, Damiano Azzinnarii ${ }^{2,4}$, Christopher R. Pryce ${ }^{2,3,4}$

5 a), Salome Kurth ${ }^{2,5, a)}$, Reto Huber ${ }^{1,2,3, \text { a), }}$

6

$7 \quad{ }^{1}$ Child Development Center, University Children's Hospital Zurich, $\mathrm{CH}-8032$ Zurich,

8 Switzerland. ${ }^{2}$ Neuroscience Center Zurich, University of Zurich and ETH Zurich, $\mathrm{CH}$ -

98057 Zurich, Switzerland. ${ }^{3}$ Zurich Center for Integrative Human Physiology,

10 University of Zurich, $\mathrm{CH}-8057$ Zurich, Switzerland. ${ }^{4}$ Preclinical Laboratory for

11 Translational Research into Affective Disorders, Department of Psychiatry,

12 Psychotherapy and Psychosomatics, Psychiatric Hospital, University of Zurich, $\mathrm{CH}$ -

138008 Zurich, Switzerland ${ }^{5}$ Baby Sleep Laboratory, Division of Pulmonology,

14 University Hospital Zurich, $\mathrm{CH}-8091$ Zurich, Switzerland

15 a) Shared last authorship

16

17 * Corresponding author and address of origin:

18 Reto Huber

19 University Children's Hospital

20 Steinwiesstr. 75

1 
218032 Zürich, SWITZERLAND

22 Reto.Huber@kispi.uzh.ch

23 Phone +41442668160

24 
ABSTRACT

26 Disturbed sleep and altered sleep homeostasis are core features of many psychiatric

27 disorders such as depression. Chronic uncontrollable stress is considered an

28 important factor in the development of depression, but little is known on how chronic

29 stress affects sleep regulation and sleep homeostasis. We therefore examined the

30 effects of chronic social stress (CSS) on sleep regulation in mice. Adult male

31 C57BL/6 mice were implanted for electrocortical recordings (ECoG) and underwent

32 either a 10-day CSS protocol or control handling (CON). Subsequently, ECoG was

33 assessed across a 24-h post-stress baseline, followed by a 4-h sleep deprivation,

34 and then a 20-h recovery period. After sleep deprivation, CSS mice showed a

35 blunted increase in sleep pressure compared to CON mice, as measured using slow

36 wave activity (SWA, electroencephalographic power between 1 - $4 \mathrm{~Hz}$ ) during non-

37 rapid eye movement (NREM) sleep. Vigilance states did not differ between CSS and

38 CON mice during post-stress baseline, sleep deprivation or recovery, with the

39 exception of CSS mice exhibiting increased REM sleep during recovery sleep.

40 Behavior during sleep deprivation was not affected by CSS. Our data provide 41 evidence that CSS alters the homeostatic regulation of sleep SWA in mice. In 42 contrast to acute social stress, which results in a faster SWA build-up, CSS 43 decelerates the homeostatic build up. These findings are discussed in relation to the 44 causal contribution of stress-induced sleep disturbance to depression. 
46 Keywords: slow wave activity; sleep regulation; depression; chronic stress; REM 47 sleep

48 


\section{INTRODUCTION}

The need for sleep - sleep pressure - is regulated homeostatically, and in the electroencephalogram is best reflected by slow wave activity (SWA, 1-4.5 Hz) during NREM sleep (EEG) [1]. Sleep pressure increases across wakefulness, further increases during sleep deprivation [2, 3], and declines in the course of naps or nighttime sleep $[4,5]$. Not only the duration but also the quality of waking, as well as the interaction with the environment, determine SWA.

In animals, including humans, the stress response to environmental stressors constitutes profound physiological changes to ensure survival and restore homeostasis [6, 7]. Environmental stressors are often acute social conflicts with conspecifics, which induce neuroendocrine and behavioral stress responses [8]. Acute social stress has been demonstrated to impact the build-up of SWA [9, 10],

61 such that a short (1 hour) social conflict with an aggressive male conspecific leads to a doubling of SWA during NREM sleep [10]. Whether this marked increase in SWA induced by social stress reflects physiological sleep or rather an unrelated stress byproduct process was investigated in a subsequent study [9]. Two groups of rats underwent a 6-hour sleep deprivation protocol, which was continuous by handling in one group and subdivided into an initial 1-hour of social conflict followed by a 5-hour

67 sleep deprivation by handling in the other group. As expected, both groups revealed

68 a strong effect of sleep deprivation by showing increased SWA during NREM sleep.

69 Interestingly, the increase was significantly greater in the "social conflict with

70 handling" group compared to the "handling only" group. Furthermore, because this 5 
71 effect of increased SWA persisted throughout extended wakefulness, the authors

72 concluded that acute social stress amplifies the homeostatic SWA build up.

73 Wakefulness duration and quality affect subsequent SWA, with a positive correlation

74 between the amount of exploratory behavior during sleep deprivation and the

75 subsequent increase in SWA ( $72 \%$ of variability in SWA explained by time spent in

76 exploratory behavior) [11]. Interestingly, both losers and winners of acute social

77 conflicts exhibit increased SWA [12]. To date, to our knowledge, there have been no

78 studies of the effects of chronic social stress (CSS) on SWA in subsequent sleep. It

79 has been demonstrated that chronic social stress results in alterations of functional

80 neural circuits [13] including resting state functional connectivity [14]. It is possible

81 that CSS affects subsequent sleep, and in either a similar or indeed different manner

82 to that reported for acute stress.

The aim of the present mouse study was to investigate the effects of CSS on

84 sleep regulation in terms of SWA. The CSS protocol was a shortened version (10

85 days rather than 15 days) of that which has been demonstrated to induce

86 depression-relevant changes in terms of resting-state functional connectivity [14],

87 reduced motivation for reward [15], increased fear learning, helplessness and

88 fatigability [16, 17], and physiological changes including increased peripheral and

89 brain inflammation $[16,17]$. Perhaps most relevant here, CSS mice exhibit increased

90 activity during the light/inactive period, as measured in terms of drinking behavior

91 [15]. Following the exposure of mice to either CSS or control handling (CON), effects

92 on sleep were examined by means of electrocortical recordings to specifically 
93

94

95

96

97

98

99

100

101

102

103

104

105

106

107

108

109

110

111

112

113 The study design is illustrated in Figure 1. Each cage contained a littermate trio of

114 C57BL/6J mice. Electrocortical surgery was conducted with two mice per trio. The 7 
115 mouse that did not undergo surgery remained in the cage and was assigned to the 116 naive (NV) group. The two implanted mice per trio were assigned to either the 117 chronic social stress (CSS) or the control (CON) group. This design was used to 118 reduce inter-group differences in baseline EEG measures. A week after surgery, 119 CSS mice were transferred to the cage of a CD-1 mouse in order to conduct the CSS 120 protocol (details below), whilst the NV and CON mice remained together as pairs. 121 Following the 10-day protocol, all mice were transferred to an electrocorticogram 122 (ECoG) laboratory. After one adaptation day, ECoG was recorded for two 123 consecutive days. The first day served as post-stress baseline, and was followed by 124 a 4-h sleep deprivation (SD) and a 20-h recovery period. Because 4-h and e.g. 6-h 125 SD induce similar SWA effects in C57BL/6 mice [18], we chose the duration of 4-h 126 SD. Whilst in the ECoG laboratory, CON and NV mice remained together in the same cage but separated by a perforated Plexiglas divider. CSS and CD-1 mice were maintained in the same cage (separated by the divider) until the end of SD, and were 129 then separated during the recovery period. The time between the last CSS attack 130 period (see 2.4) and the start of the baseline recording was $74-77 \mathrm{~h}$.

\section{$132 \quad 2.3$ Surgical procedure}

133 Surgery for ECoG recording was conducted according to a standard procedure [19]. 134 Under sevoflurane (nose mask, 3-6 \%; 1:4 $\mathrm{O}_{2}$-to-air ratio), mice were implanted on 135 the dura with three gold-plated miniature screws $(\varnothing=0.9 \mathrm{~mm})$ using the following 136 coordinates: right hemisphere, frontal, $1.5 \mathrm{~mm}$ anterior to bregma, $2 \mathrm{~mm}$ lateral to 137 midline; right hemisphere, parietal, $2 \mathrm{~mm}$ anterior to lambda, $2 \mathrm{~mm}$ lateral to midline; 
138 midline, reference, above cerebellum, $2 \mathrm{~mm}$ posterior to lambda. Two gold wires $(\varnothing$ $139=0.2 \mathrm{~mm}$ ) were inserted bilaterally into the neck muscles for electromyogram (EMG) 140 recording. Electrodes were attached to stainless steel wires connected to the skull 141 with dental acrylic cement. Mice received one pre- and two post-operative doses of 142 analgesic (0.1 mg/kg buprenorphine, s.c.). Mice regained pre-surgery weight within 143 2-3 days and were given a 7-day recovery period before the CSS protocol started 144 (Fig. 1). At the end of the experiment all brains were inspected for potential infections 145 from electrodes and observed to be free of cortical damage.

$147 \quad 2.4$ Chronic social stress (CSS)

148 The study protocol was adapted from that which we have developed for the study of 149 neural, behavioral and physiological effects of CSS in mice (e.g., [14, 16]). The 150 protocol is usually applied for 15 days. In the present study we were concerned that 151 the daily attack sessions could result in damage to the ECoG cap. Accordingly, we 152 reduced the number of daily attack sessions to 10 per CSS mouse (Fig. 1). On day 1 153 of the CSS protocol, each CSS mouse was placed in the cage of an aggressive CD-1 154 mouse so that bouts of attack occurred. The mice remained in the same cage for a 155 maximum of either $60 \mathrm{sec}$ of attack or $10 \mathrm{~min}$. A transparent Plexiglas divider was 156 then used to separate the mice in the same cage. The divider was perforated to allow 157 sensory communication while preventing physical attacks. After $24 \mathrm{~h}$ each CSS 158 mouse was placed in the cage of a novel CD-1 aggressor mouse, and the divider 159 was removed for the 10-min attack period. Each day mouse pairs were interchanged 9 
160 in this way and the procedure repeated across 10 consecutive days. Physical attack

161 sessions were conducted between $14 \mathrm{~h}$ and $17 \mathrm{~h}$. Overall, the shortest daily 162 cumulative attack duration was $26 \mathrm{~s}$ and the $60 \mathrm{~s}$ maximum duration occurred 163 frequently. Across the eight CSS mice the mean daily cumulative duration of attack 164 time was consistent: from $42.1 \pm 4.2 \mathrm{~s}$ to $46.9 \pm 4.5 \mathrm{~s}$ (Mean $\pm \mathrm{SE}$ ). Bite wounds were 165 prevented by trimming the incisor teeth of the CD-1 mice every third day using rodent 166 tooth-cutting forceps. Mice did not obtain major visible injuries. Based on subsequent 167 poor signal quality, the ECoG cap of one CSS mouse was damaged (see Results). 168 CON and NV mice were handled daily. All mice were weighed daily: CSS typically 169 induces an increase in the day-to-day change in body weight $(\Delta \mathrm{BW})[14,16,17]$. We 170 calculated $\Delta \mathrm{BW}$ for 5 -day time blocks of pre-CSS (day -4 to 1 ), CSS day 2 to 6 and 171 CSS day 7 to 11 as $\Delta B W=($ abs (BW day $n-B W$ day $n-1)) /(B W \text { day } n-1)^{*} 100$.

\subsection{Electrocortical recordings (ECoG)}

174 During ECoG recordings, a divider separated CSS mice from aggressor CD-1 mice, 175 and likewise a divider separated CON mice from NV littermates. The ECoG cap was 176 attached to a connector soldered to a thin cable attached to a swivel. The light-dark 177 cycle was maintained throughout the experiment (light on 19:00-07:00 h), and food 178 and water were available ad libitum. Before each recording, EEG and EMG channels 179 were calibrated with a $10 \mathrm{~Hz}, 300-\mu \mathrm{V}$ peak-to-peak sine wave. ECoG and EMG 180 signals were amplified (amplification factor 2000), filtered (high-pass filter: $-3 \mathrm{~dB}$ at $1810.016 \mathrm{~Hz}$; low-pass filter: $-3 \mathrm{~dB}$ at $40 \mathrm{~Hz}$ ), sampled with $512 \mathrm{~Hz}$, digitally filtered 182 (ECoG: low-pass finite impulse response (FIR filter, $25 \mathrm{~Hz}$; EMG: band-pass FIR 10 
183 filter, $20-50 \mathrm{~Hz})$ ), and stored with a resolution of $128 \mathrm{~Hz}$. ECoG power spectra were 184 computed for $4 \mathrm{~s}$ epochs by a fast Fourier transform routine. Adjacent $0.25 \mathrm{~Hz}$ 185 frequency bins were averaged into $0.5 \mathrm{~Hz}(0.25-5 \mathrm{~Hz})$ and $1.0 \mathrm{~Hz}(5.25-25 \mathrm{~Hz})$ 186 bins. SWA was computed within $1.0-4.0 \mathrm{~Hz}$.

187 The vigilance states NREM sleep, REM sleep and Wake were determined 188 visually off-line from the ECoG and EMG signals, according to standard methods 189 [19]. The percentage of epochs containing artefacts were: for NREM sleep: $6.9 \pm 3.1$ $190 \%$ in CON, $1.5 \pm 0.4 \%$ in CSS during post-stress baseline, $3.7 \pm 2.8 \%$ in CON, $1.6 \pm$ $1910.5 \%$ in CSS during recovery sleep; for REM sleep: $1.7 \pm 0.6 \%$ in CON, $0.5 \pm 0.2 \%$ 192 in CSS during post-stress baseline, $0.9 \pm 0.6 \%$ in CON, $0.6 \pm 0.2 \%$ in CSS during 193 recovery sleep; and for wake: $31.6 \pm 4.1 \%$ in CON, $30.4 \pm 3.7 \%$ in CSS during post194 stress baseline, $37.7 \pm 3.9 \%$ in CON, $33.9 \pm 3.4 \%$ in CSS during recovery sleep (M $195 \pm$ SE). Epochs containing artefacts were excluded from spectral analysis.

2.6 Sleep deprivation

198 As reported in previous studies [18], sleep deprivation (SD) was performed for 4-h, 199 starting at light onset $(19: 00 \mathrm{~h})$ on the second day after transfer to the ECoG 200 laboratory, by means of introducing new objects to the cage. Mice were videotaped 201 during SD (Roline, RIC-45 IP Camera). Based on these video recordings behavior 202 was then scored off-line from an ethogram, using instantaneous sampling with a 10203 sec interval. The mutually exclusive behaviors scored were "no behavior", "quietly 204 awake", "grooming", "exploring", "object exploring" and "social interaction at the 11 
205 divider". "Number of rearings" during exploratory behavior was also identified [20]. All

206 sensory stimulations undertaken by the experimenter to restrict mice from sleep, i.e., 207 “disturb", “object in”, “object out”, were also counted. The experimenter who scored 208 behaviors as well as ECoG was blind to the experimental condition.

$210 \quad 2.7$ Statistical analysis

211 The frontal derivation was analysed. ECoG data analysis and statistics were 212 performed using the MATLAB software package (MathWorks). Analysis of variance 213 (ANOVA, mixed model) was conducted with the between-subject factor of 'group' 214 (CSS, CON) and, depending on the dependent variable, a within-subject factor of 215 'time' e.g., day blocks for body weight, 2-h blocks for vigilance states and SWA. In 216 the case of significant interaction effects, post hoc testing was carried out using the 217 least significant difference (LSD) test. Statistical significance was set at $p<0.05$ and $218 p<0.1$ was used as a borderline non-significant trend effect. Data are expressed as 219 mean +/- standard deviation or standard error, as indicated.

\section{$221 \quad 3$. RESULTS}

222 The present study investigated the effects of a 10-day CSS protocol on vigilance 223 states and on SWA as a marker of the homeostatic regulation of sleep in mice; the 224 CSS protocol was adapted for ECoG recordings. In line with previous CSS studies, 225 mean duration of total daily attack was $44.5 \pm 1.9 \mathrm{sec}$ per mouse. For absolute BW 226 (Fig. 2A), there was a significant 'group' $x$ 'time' interaction $(F(4,40)=4.883, p<$ 227 0.003), but no significant group difference at any specific time point $(p \geq 0.06)$; the 12 
228 significant main effect of 'time' $(p<0.0005)$ reflected the increase in BW across time 229 blocks. For day-to-day $\Delta \mathrm{BW}$ (Fig. 2B), in accordance with previous studies, there was 230 a significant 'group' $x$ 'time' interaction $(F(4,40)=9.552, p<0.0005)$, with post hoc 231 analysis showing increased $\triangle \mathrm{BW}$ in CSS relative to CON and NV mice for days 2-6 $232(p<0.0005)$ and in CSS relative to NV mice for days $7-11(p<0.02)$ (Figure 2 about here)

234

$235 \quad 3.1$ Vigilance states

236 Following a one-day adaptation to the ECoG laboratory, sleep was assessed during 237 a 24-h post-stress baseline, a 4-h SD and a 20-h recovery period. One CSS mouse 238 was excluded from the study because of poor signal quality, resulting in $n=7$ for CSS 239 and $n=8$ for CON. During post-stress baseline recording, CSS and CON mice 240 exhibited vigilance states typical for light-dark conditions, with a predominance of 241 Wake during the dark period and a predominance of NREM sleep during the light 242 period (Fig. 3, p $<0.0001$ for factor 'time'). As expected, SD markedly increased 243 Wake $(99.4 \pm 0.3 \%$ hours $1-2$ and $98.4 \pm 0.7 \%$ hours $3-4$ in CON mice, $99.3 \pm 0.3 \%$ 244 hours 1-2 and $97.0 \pm 1.1 \%$ hours $3-4$ in CSS mice; Fig. 3A). There was no significant 245 effect of CSS in post-stress baseline or recovery for Wake (Fig. 3A) or in post-stress 246 baseline or recovery for NREM sleep (Fig. 3B) (all 'group' effects, $p=$ n.s.). For REM 247 sleep, while no group effect was found during post-stress baseline ( $p=$ n.s.), a 248 difference was found in recovery, with CSS mice showing significantly increased 249 REM sleep relative to CON mice ('group' $F(1,9)=5.2, p<0.05$; Fig. $3 C$ ). In order to 13 
250 examine the effect of SD on vigilance states, we conducted a 2-way ANOVA with

251 factors 'condition' (post-stress baseline vs. recovery) and 'time' (hour 5-6 to hour 22252 24) and included CSS and CON mice. For NREM sleep (Fig. 3B), there was a main 253 effect of 'condition' $(p<0.05)$ reflecting increased NREM sleep after SD that was 254 sustained across the $18 \mathrm{~h}$ period. This was also the case for REM sleep, with a main 255 effect of 'condition' ( $p<0.05)$ (Fig. 3C).

3.2 Slow-wave activity

259 We next investigated CSS effects on the homeostatic regulation of sleep in terms of 260 SWA. Under post-stress baseline conditions, SWA followed a similar time course in 261 both groups (Fig. 4A, $p=$ n.s. for factor 'group'). As expected, SD induced a SWA 262 increase in both groups (comparison with post-stress baseline, $p<0.05$, strongest 263 effect initially). For 'group' $\mathrm{x}$ 'time' ANOVA of the recovery period there was a 264 significant interaction effect on SWA $(F(1,9)=2.3$, $p<0.05$; Fig. 5B). Post hoc testing 265 identified that the SWA increase was smaller in CSS mice relative to CON mice at 266 the 2-h time intervals R1 (trend-level, $p=0.06$ ), R2 (trend-level, $p=0.09$ ) and R3 ( $p$ $267<0.05)$. At each of these time intervals we then conducted an a posteriori power 268 spectral analysis of CSS effects. In a 'group' $x$ 'frequency' ANOVA, there was a 269 significant main effect of 'group' for R1 $(p<0.007)$ and significant interaction effects 270 for $R 2(p<0.00001)$ and R3 $(p<0.00001)$. For each time interval, post hoc LSD 271 tests revealed reduced EEG power in CSS mice compared with CON mice specific to 272 low frequencies i.e., the SWA frequency band, but extending up to $8 \mathrm{~Hz}$ in interval R1 14 
273 (Fig. 5A-C). Therefore, power spectral analysis confirmed that the group effect was 274 most prominent when sleep pressure was high. Because SWA depends on wake 275 quality [11], we then examined for associations with the scores for behaviors 276 measured during SD. CSS and CON mice exhibited no differences on any scored 277 behavior $(p \geq 0.15)$. The number of interventions during SD did not differ between 278 groups, as quantified by 'number of disturbances' (CSS: $3.1 \pm 2.1$, CON: $5.8 \pm 9.6, p$ $279=$ n.s.) and 'times object in/out' (CSS: $3.6 \pm 3.4$, CON: $6.9 \pm 10.4, p=$ n.s.). These 280 data indicate that the changes in SWA do not appear to be explicable in terms of a 281 difference in the quality of waking during SD.

\section{DISCUSSION}

286 This study investigated the effects of chronic social stress on the homeostatic 287 regulation of sleep in mice. While CSS did not change SWA during a 24-h post-stress 288 baseline, $4 \mathrm{~h}$ of SD elicited a blunted homeostatic SWA increase during subsequent 289 sleep and briefly increased REM sleep. These findings show an effect of CSS on 290 sleep homeostasis specific to the condition of enforced wakefulness, with no CSS 291 effect apparent under baseline conditions. Our data show a SWA increase in CSS 292 and CON mice following sleep deprivation. However, this SWA increase was 293 significantly smaller in CSS relative to CON mice in a $6 \mathrm{~h}$ period following SD. That 294 CSS mice did not show attenuated SWA at the beginning of post-stress baseline 15 
295 sleep indicating that a sleep challenge is needed to uncover effects of CSS.

296 Therefore, chronic stress together with the additional pressure of waking results in 297 altered sleep physiology. We cannot exclude the possibility that a longer stress 298 exposure protocol, including the 15-day protocol that we have used in other studies 299 e.g. [14], would also impact on SWA in baseline sleep. Interestingly, CSS mice also 300 showed increased REM sleep during recovery sleep, supporting an association 301 between SWA in NREM sleep and features of REM sleep, as reported previously $302[21]$

Previous research revealed that acute $(1 \mathrm{~h})$ social conflict in rats leads to 304 increased SWA in subsequent sleep [10], whereas the current study provides 305 evidence that chronic (10 day) social stress leads to an attenuated increase in SWA 306 following SD. Therefore, the effects of social stress on sleep homeostasis would 307 appear to depend on the duration of social stress. CSS may induce specific changes 308 at a neurophysiological level that are reflected in altered SWA. Similar to our results, 309 in rats, Mrdalj et al. demonstrated that chronic postnatal stress flattened the sleep 310 homeostasis curve in a long-lasting manner [22]. Together, these studies suggest 311 that stress affects the most established marker of sleep homeostasis, SWA, and that 312 the direction of the effect is dependent on the duration of stress exposure. A further 313 finding is that whereas acute stress effects were reported to be specific to SWA [10], 314 CSS affects an extended frequency range up to $8 \mathrm{~Hz}$ and the study of chronic stress 315 in rats also reported this [22]. When comparing the current findings with the existing 316 literature, it is important to consider that the present study did not investigate the 317 response to social conflict per se, but rather investigated the effects of chronic social 16 
318 stress in terms of brief conflict encounters with and otherwise continuous distal 319 exposure to dominant, aggressive mice.

The daily attacks that form part of CSS took place at the same time each day

321 (i.e., $14-17 \mathrm{~h}$ ) across the 10 days. Predictability can influence the perception of the 322 stressor and modulate the direction of the effects of stress on sleep [23]. Predictable 323 stressors may be perceived as less severe, which may contribute to the absence of 324 group differences in the post-stress baseline. The efficacy of our protocol compared 325 to the 15-day protocol which leads to depression-relevant effects on physiology, brain 326 and behavior (e.g., [15, 16, 24]), was demonstrated by the consistent biomarker of 327 increased body weight delta in CSS compared with CON mice. Quantifying the 328 neurotransmitter and neuromodulators related to stress in addition to sleep (e.g., 329 monoamines, hypocretin/orexin, corticotropin releasing hormone, inflammatory 330 factors) would provide a more mechanistic underpinning of the physiological effects 331 of CSS. Indeed, transcriptomic studies of cortical and limbic brain regions have 332 identified altered expression of a number of genes relevant to dopamine and 333 serotonin neurotransmission in 15-day CSS mice [16].

334 Chronic psychosocial stress is a major aetiological factor in depression [25, 335 26]. The S-deficiency hypothesis of Borbély et al. [27] proposes a slower build-up of 336 homeostatic sleep pressure during wakefulness in depressed patients, including 337 lower SWA at sleep onset. Indeed, depressed humans show a slower accumulation 338 of SWA [28-30], and the current findings are in line with this. CSS induces adrenal 339 gland and spleen hypertrophy, and increased levels of pro-inflammatory factors in the 17 
340 periphery and brain [16, 17], indicating an impact of CSS on stress-responsive 341 systems that, in turn, are associated with altered sleep [31].

As expected, effects of SD on vigilance states included an increase in NREM 343 and REM sleep, and in CSS and CON mice. Changes in vigilance states could have 344 accounted for the post-SD blunted SWA increase in CSS mice. However, with the 345 exception of increased REM sleep during the recovery period, vigilance states did not 346 differ in CSS compared to CON mice. We thus conclude that CSS in mice induces 347 specific changes in the best-established marker of sleep regulation, namely SWA. 348 Interestingly, in a recent study we observed that CSS mice (15-day protocol) exhibit 349 increased light-phase activity compared with CON mice [15]. SWA is determined by 350 the quality as well as the quantity of wakefulness, e.g. [11], such that differences in 351 behavior and therefore wakefulness quality during SD could have accounted for the 352 observed CSS-effect. However, results showed no behavioral differences between 353 CSS and CON mice during the SD, suggesting that neither alterations in vigilance 354 nor behavioral differences during SD contributed to the blunted SWA increase in 355 CSS mice.

$356 \quad$ Using 4-day stress exposure in the form of repeated fear conditioning of an 357 auditory tone to footshock, Sanford et al. report a subsequent reduction in REM 358 sleep, and re-exposure to the tone several days later again reduced REM sleep [32]. 359 Accordingly, physical and social stressors, as well as the stress duration, would 360 appear to contribute to effects on REM. ECoG-assessments will be needed to 361 measure CSS-dependent changes in vigilance states long-term. Additionally, future 362 studies should investigate topographical differences in the effect of CSS exposure on 18 
363 sleep regulation by also analysing EEG from the parietal derivation. Future studies

364 may also examine whether chronic stress affects specific frequency ranges in waking 365 and in REM sleep.

The study of neuronal correlates of SWA might well provide mechanistic 367 insight into impaired homeostatic regulation in CSS. SWA closely reflects 368 synchronized activity of cortical neurons [33] and synaptic plasticity [34]. The blunted build-up of SWA in CSS mice might thus indicate adaptations to chronic stress by means of impaired synchronisation via changes in synaptic mechanisms. Synaptic

371 plasticity is linked to stress via endocrine and immune pathways [16, 35-37]. It is 372 possible that CSS is associated with altered pyramidal cell excitability and inhibitory 373 activity [37], via stress-related changes of postsynaptic AMPA, NMDA and glutamate 374 receptors in the medial prefrontal cortex. Interestingly, CSS results in increased 375 functional connectivity within prefrontal cortex [14] and in changes in transcriptome 376 expression [16].

377 In summary, our findings show that CSS alters the homeostatic SWA 378 response, and does so in a direction opposite to that induced by acute stress. It will 379 be important to further increase the understanding of interactions between chronic 380 social stress, sleep regulation, and physiology-behavior links. While quality of waking 381 can alter sleep regulation and vigilance states, quality of sleep may increment the 382 development of a depressive phenotype [38], suggesting a bi-directional and 383 probably self-intensifying cycle. 


\section{ACKNOWLEDGEMENTS}

386 We thank Peter Meerlo for helpful comments and suggestions, Hannes Sigrist for 387 technical support and Björn Henz for animal care. This work was supported by the 388 Neuroscience Center Zurich, the Swiss National Science Foundation (PP00A389 114923, PP00P3-13538 and 31003A-141137) and the Clinical Research Priority 390 Programme (CRPP) "Sleep and Health".

391

392 FINANCIAL DISCLOSURES

393 All authors declare no competing financial interests.

394 
396 [1] A.A. Borbely, A two process model of sleep regulation, Hum Neurobiol 1(3) (1982) 195204.

398 [2] A.A. Borbely, I. Tobler, M. Hanagasioglu, Effect of sleep deprivation on sleep and EEG power spectra in the rat, Behav Brain Res 14(3) (1984) 171-82.

[3] P. Franken, D. Chollet, M. Tafti, The homeostatic regulation of sleep need is under genetic control, J Neurosci 21(8) (2001) 2610-21.

[4] I. Karacan, R.L. Williams, W.W. Finley, C.J. Hursch, The effects of naps on nocturnal sleep: influence on the need for stage-1 REM and stage 4 sleep, Biol Psychiatry 2(4) (1970) 391-9.

[5] E. Werth, D.J. Dijk, P. Achermann, A.A. Borbely, Dynamics of the sleep EEG after an early evening nap: experimental data and simulations, Am J Physiol 271(3 Pt 2) (1996) R501-10.

[6] B.S. McEwen, J.C. Wingfield, What is in a name? Integrating homeostasis, allostasis and stress, Horm Behav 57(2) (2010) 105-11.

410 [7] J.M. Koolhaas, A. Bartolomucci, B. Buwalda, S.F. de Boer, G. Flugge, S.M. Korte, P. G. van Dijk, M. Wohr, E. Fuchs, Stress revisited: a critical evaluation of the stress concept, Neurosci Biobehav Rev 35(5) (2011) 1291-301.

[8] J.M. Koolhaas, P. Meerlo, S.F. De Boer, J.H. Strubbe, B. Bohus, The temporal dynamics of the stress response, Neuroscience and biobehavioral reviews 21(6) (1997) 775-82.

[9] P. Meerlo, E.A. de Bruin, A.M. Strijkstra, S. Daan, A social conflict increases EEG slowwave activity during subsequent sleep, Physiol Behav 73(3) (2001) 331-5.

[10] P. Meerlo, B.J. Pragt, S. Daan, Social stress induces high intensity sleep in rats, Neurosci Lett 225(1) (1997) 41-4.

[11] R. Huber, G. Tononi, C. Cirelli, Exploratory behavior, cortical BDNF expression, and sleep homeostasis, Sleep 30(2) (2007) 129-139.

[12] J. Kamphuis, M. Lancel, J.M. Koolhaas, P. Meerlo, Deep sleep after social stress: NREM sleep slow-wave activity is enhanced in both winners and losers of a conflict, Brain Behav Immun 47 (2015) 149-54.

[13] R.C. Bagot, H.M. Cates, I. Purushothaman, Z.S. Lorsch, D.M. Walker, J. Wang, X. Huang, O.M. Schluter, I. Maze, C.J. Pena, E.A. Heller, O. Issler, M. Wang, W.M. Song, J.L. Stein, X. Liu, M.A. Doyle, K.N. Scobie, H.S. Sun, R.L. Neve, D. Geschwind, Y. Dong, L. Shen, B. Zhang, E.J. Nestler, Circuit-wide Transcriptional Profiling Reveals Brain RegionSpecific Gene Networks Regulating Depression Susceptibility, Neuron 90(5) (2016) 969-83. [14] J. Grandjean, D. Azzinnari, A. Seuwen, H. Sigrist, E. Seifritz, C.R. Pryce, M. Rudin, Chronic psychosocial stress in mice leads to changes in brain functional connectivity and metabolite levels comparable to human depression, Neurolmage (2016).

[15] G. Bergamini, F. Cathomas, S. Auer, H. Sigrist, E. Seifritz, M. Patterson, C. Gabriel, C.R. Pryce, Mouse psychosocial stress reduces motivation and cognitive function in operant reward tests: A model for reward pathology with effects of agomelatine, Eur Neuropsychopharmacol 26(9) (2016) 1448-64.

[16] D. Azzinnari, H. Sigrist, S. Staehli, R. Palme, T. Hildebrandt, G. Leparc, B. Hengerer, E. Seifritz, C.R. Pryce, Mouse social stress induces increased fear conditioning, helplessness and fatigue to physical challenge together with markers of altered immune and dopamine function, Neuropharmacology 85 (2014) 328-41. 
[17] R. Fuertig, D. Azzinnari, G. Bergamini, F. Cathomas, H. Sigrist, E. Seifritz, S. Vavassori, A. Luippold, B. Hengerer, A. Ceci, C.R. Pryce, Mouse chronic social stress increases blood and brain kynurenine pathway activity and fear behaviour: Both effects are reversed by inhibition of indoleamine 2,3-dioxygenase, Brain Behav Immun 54 (2016) 59-72.

[18] R. Huber, T. Deboer, I. Tobler, Effects of sleep deprivation on sleep and sleep EEG in three mouse strains: empirical data and simulations, Brain Res 857(1-2) (2000) 8-19.

[19] P. Franken, D.J. Dijk, I. Tobler, A.A. Borbely, Sleep deprivation in rats: effects on EEG power spectra, vigilance states, and cortical temperature, Am J Physiol 261(1 Pt 2) (1991) R198-208.

[20] W.E. Crusio, Genetic dissection of mouse exploratory behaviour, Behav Brain Res 125(1-2) (2001) 127-32.

[21] D.G. Beersma, P. Achermann, Changes of sleep EEG slow-wave activity in response to sleep manipulations: to what extent are they related to changes in REM sleep latency?, J Sleep Res 4(1) (1995) 23-29.

[22] J. Mrdalj, S. Pallesen, A.M. Milde, F.K. Jellestad, R. Murison, R. Ursin, B. Bjorvatn, J. Gronli, Early and later life stress alter brain activity and sleep in rats, PLoS One 8(7) (2013) e69923.

[23] L.D. Sanford, D. Suchecki, P. Meerlo, Stress, arousal, and sleep, Current topics in behavioral neurosciences 25 (2015) 379-410.

[24] J. Grandjean, D. Azzinnari, A. Seuwen, H. Sigrist, E. Seifritz, C.R. Pryce, M. Rudin, Chronic psychosocial stress in mice leads to changes in brain functional connectivity and metabolite levels comparable to human depression, Neuroimage 142 (2016) 544-552.

[25] K.S. Kendler, L.M. Karkowski, C.A. Prescott, Causal relationship between stressful life events and the onset of major depression, Am J Psychiatry 156(6) (1999) 837-41.

[26] C.R. Pryce, E. Fuchs, Chronic psychosocial stressors in adulthood: Studies in mice, rats and tree shrews, Neurobiology of Stress (in press).

[27] A.A. Borbely, The S-deficiency hypothesis of depression and the two-process model of sleep regulation, Pharmacopsychiatry 20(1) (1987) 23-9.

[28] A.A. Borbely, I. Tobler, M. Loepfe, D.J. Kupfer, R.F. Ulrich, V. Grochocinski, J. Doman, G. Matthews, All-night spectral analysis of the sleep EEG in untreated depressives and normal controls, Psychiatry Res 12(1) (1984) 27-33.

[29] R. Hoffmann, W. Hendrickse, A.J. Rush, R. Armitage, Slow-wave activity during nonREM sleep in men with schizophrenia and major depressive disorders, Psychiatry Res 95(3) (2000) 215-25.

[30] D.T. Plante, E.C. Landsness, M.J. Peterson, M.R. Goldstein, T. Wanger, J.J. Guokas, G. Tononi, R.M. Benca, Altered slow wave activity in major depressive disorder with hypersomnia: a high density EEG pilot study, Psychiatry Res 201(3) (2012) 240-4.

[31] J.M. Krueger, J.A. Majde, Humoral links between sleep and the immune system: research issues, Ann N Y Acad Sci 992 (2003) 9-20.

[32] L.D. Sanford, X. Tang, R.J. Ross, A.R. Morrison, Influence of shock training and explicit fear-conditioned cues on sleep architecture in mice: strain comparison, Behavior genetics 33(1) (2003) 43-58.

[33] V.V. Vyazovskiy, U. Olcese, Y.M. Lazimy, U. Faraguna, S.K. Esser, J.C. Williams, C. Cirelli, G. Tononi, Cortical firing and sleep homeostasis, Neuron 63(6) (2009) 865-78.

[34] G. Tononi, C. Cirelli, Sleep and the price of plasticity: from synaptic and cellular homeostasis to memory consolidation and integration, Neuron 81(1) (2014) 12-34.

[35] M.F. Dallman, S.F. Akana, C.S. Cascio, D.N. Darlington, L. Jacobson, N. Levin, Regulation of ACTH secretion: variations on a theme of $B$, Recent progress in hormone research 43 (1987) 113-73. 
490 [36] M.F. Dallman, M.T. Jones, Corticosteroid feedback control of ACTH secretion: effect of 491 stress-induced corticosterone ssecretion on subsequent stress responses in the rat, 492 Endocrinology 92(5) (1973) 1367-75.

493 [37] B. Myers, J.M. McKlveen, J.P. Herman, Glucocorticoid actions on synapses, circuits, 494 and behavior: implications for the energetics of stress, Front Neuroendocrinol 35(2) (2014) $495 \quad 180-96$.

496 [38] P. Meerlo, R. Havekes, A. Steiger, Chronically restricted or disrupted sleep as a causal 497 factor in the development of depression, Current topics in behavioral neurosciences 25 498 (2015) 459-81. 
FIGURE LEGENDS

502

503 Figure 1: Overview of the study design. Groups are represented by shaded 504 backgrounds (black: chronic social stress (CSS); grey: control (CON); white: naive 505 (NV)). Experiment refers to CSS protocol (CSS group) or handling (CON and NV 506 groups); ECoG: electrocortical recording. Numbers of animals included in analysis 507 are indicated for each group.

508

509 Figure 2: Body weight from immediately prior to until the end of the chronic social 510 stress period. (A) Absolute body weight. (B) Day-to-day body weight delta. In mixed511 model ANOVA there was a 'group' x 'time' interaction with post hoc tests identifying 512 increased $\triangle B W$ in CSS mice vs CON and NV mice at days $2-6\left(^{* \star * *} p<0.0005\right)$ and 513 in CSS mice vs NV mice at days $7-11\left({ }^{*} p<0.02\right)$.

514

515 Figure 3: Vigilance states during post-stress baseline (24 h), sleep deprivation (4 h) 516 and recovery $(20 \mathrm{~h})$. White and black bars represent 12-h light and 12-h dark periods, 517 respectively. (A) Wake, (B) non-rapid eye movement (NREM), and (C) rapid eye 518 movement (REM) sleep are shown for chronic social stress (CSS) and control (CON) 519 groups. Data are averaged across 2-h time intervals. Values are expressed as a 520 percentage of entire recording time. A mixed model ANOVA was performed for each 521 panel with between-factor 'group' (CON vs. CSS) and within-factor 'time' (12 $\times 2 \mathrm{~h}$ for 522 Post-Stress Baseline, $10 \times 2 \mathrm{~h}$ for Recovery). REM sleep in recovery was 
523 significantly increased in CSS relative to CON mice (mixed model ANOVA and post

524 hoc LSD tests are indicated as text in the figure).

526 Figure 4: Slow wave activity (SWA, 1-4.5 Hz) in chronic social stress (CSS) and 527 control $(\mathrm{CON})$ groups during $(\mathrm{A})$ post-stress baseline and $(\mathrm{B})$ recovery. White and 528 black bars represent 12-h light and 12-h dark periods, respectively. SWA is averaged 529 across 2-h time intervals. Values are expressed as a percentage of the mean of 24-h 530 post-stress baseline SWA. Asterisks (gray box) indicate time intervals with significant 531 or trend-level increases in SWA relative to the post-stress baseline condition for CON 532 (black) and CSS (red) mice (“* " $p<0.05$; " o" $p<0.1$; i.e., 6 h post-stress baseline vs. $5336 \mathrm{~h}$ recovery etc). For post-stress baseline and recovery separately, ANOVA was 534 performed with 'group' (CSS vs. CON) as between-subject factor and 'time' (2-h 535 intervals) as within-subject factor. For post-stress baseline there was a significant 536 effect of 'time' $(F(1,11)=2.7, p<0.005)$ and no 'group' or interaction effect (both $p=$ 537 n.s.). For recovery, there was a significant 'group' $x$ 'time' interaction $(F(1,9)=2.3, p$ $538<0.05)$, reflecting group differences in SWA. Post-hoc LSD tests identified lower 539 SWA in CSS compared to CON mice in the first three $2 \mathrm{~h}$ intervals post-SD (indicated 540 as R1, R2, R3).

542 Figure 5: Power spectral analysis of electrocortical recordings (ECoG) after sleep 543 deprivation. Power spectra are shown for chronic social stress (CSS, red) and control 544 (CON, black) mice for (A) hours 1-2 (R1), (B) hours 3-4 (R2), and (C) hours 5-6 (R3) 25 
545 post-sleep deprivation, expressed as a percentage of 24-h post-stress baselines $(M+$ 546 SE). Mixed-model ANOVA with between-subject factor 'group' and within-subject 547 factor 'frequency' revealed for $\mathrm{R} 1$ : 'group' $F(1,29)=10.3, p=0.007$, 'interaction' $548 F(1,29)=1.0, p=$ n.s.; for $R 2$ 'group' $F(1,29)=50.4, p<0.00001$, 'interaction' $F(1,29)$ $549=11.7, p<0.00001$; and for R3: 'group' $F(1,29)=41.2, p=0.00002$, 'interaction' $550 F(1,29)=27.4, p<0.00001$. Post hoc LSD tests identified frequency-specific group 551 effects $\left({ }^{*} p<0.05\right)$. “

552

553 


\section{FIGURES}

555

556 Figure 1:

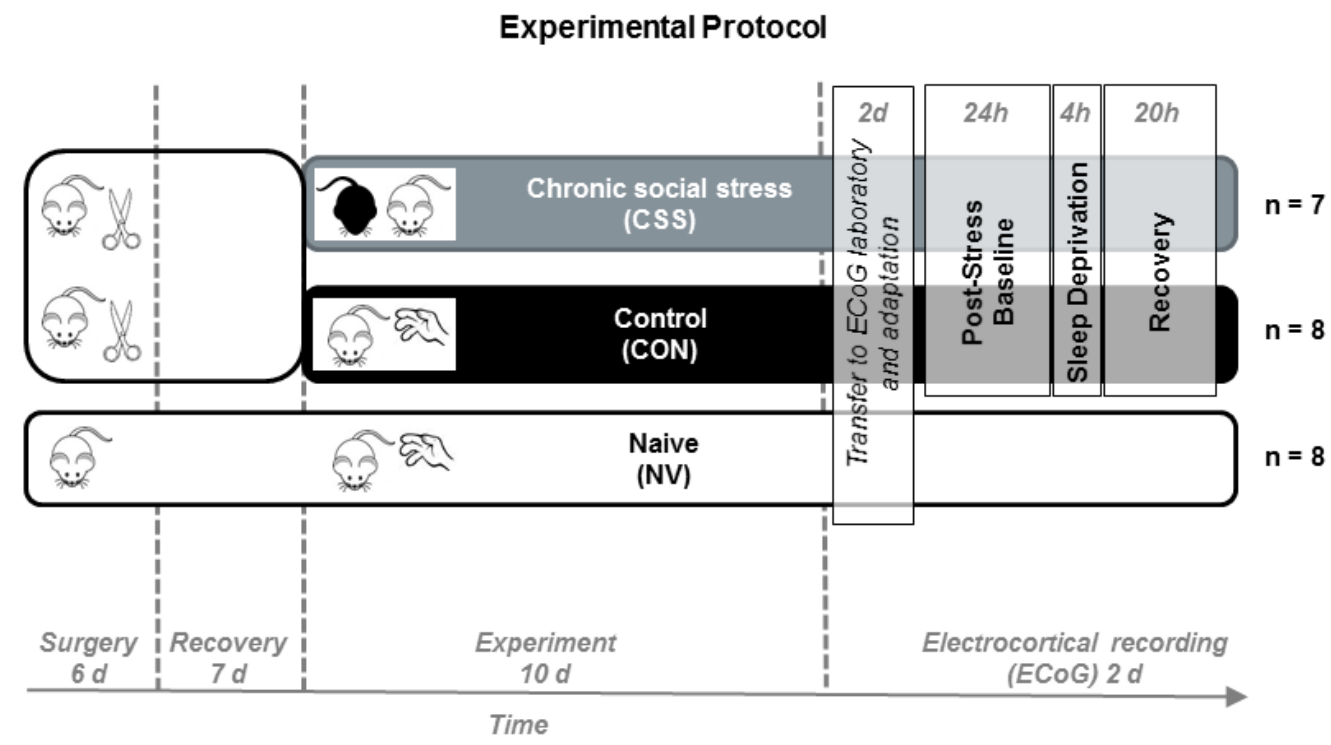

557

558

559

560 
561 Figure 2:

A

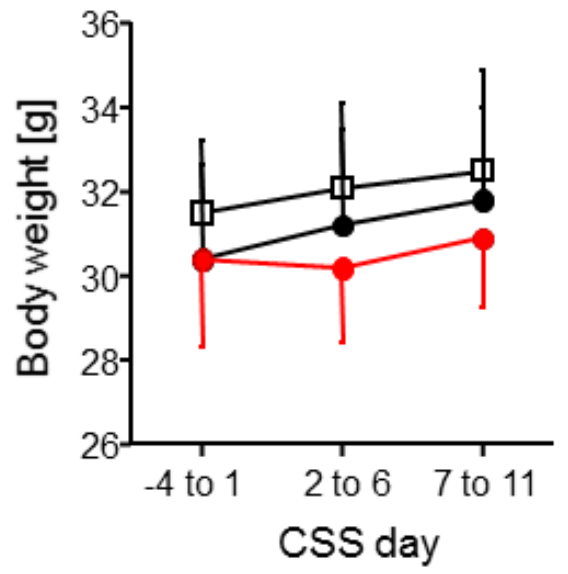

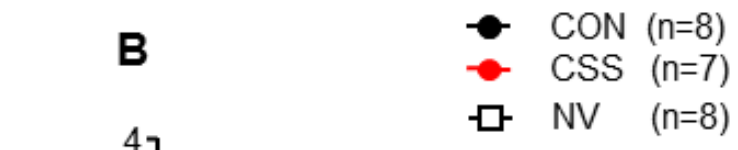

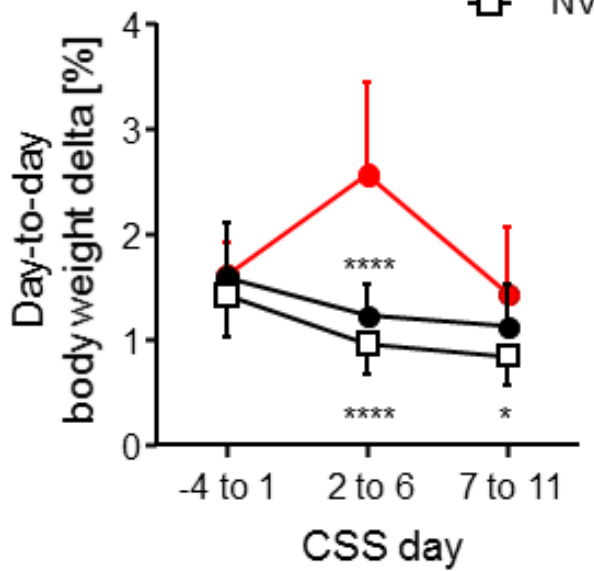

563 
Figure 3:

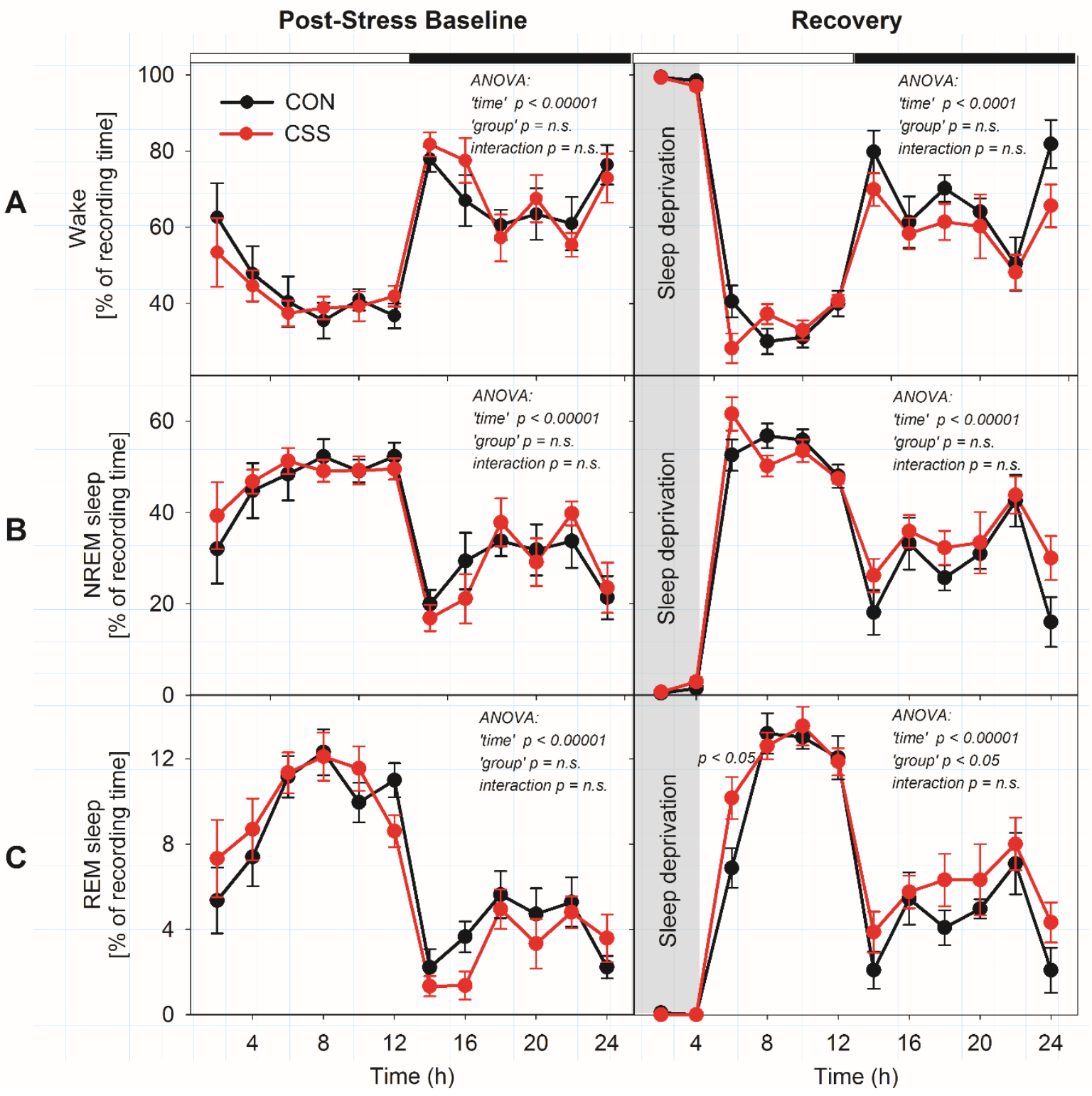

566

567

568

569 
570 Figure 4:

A

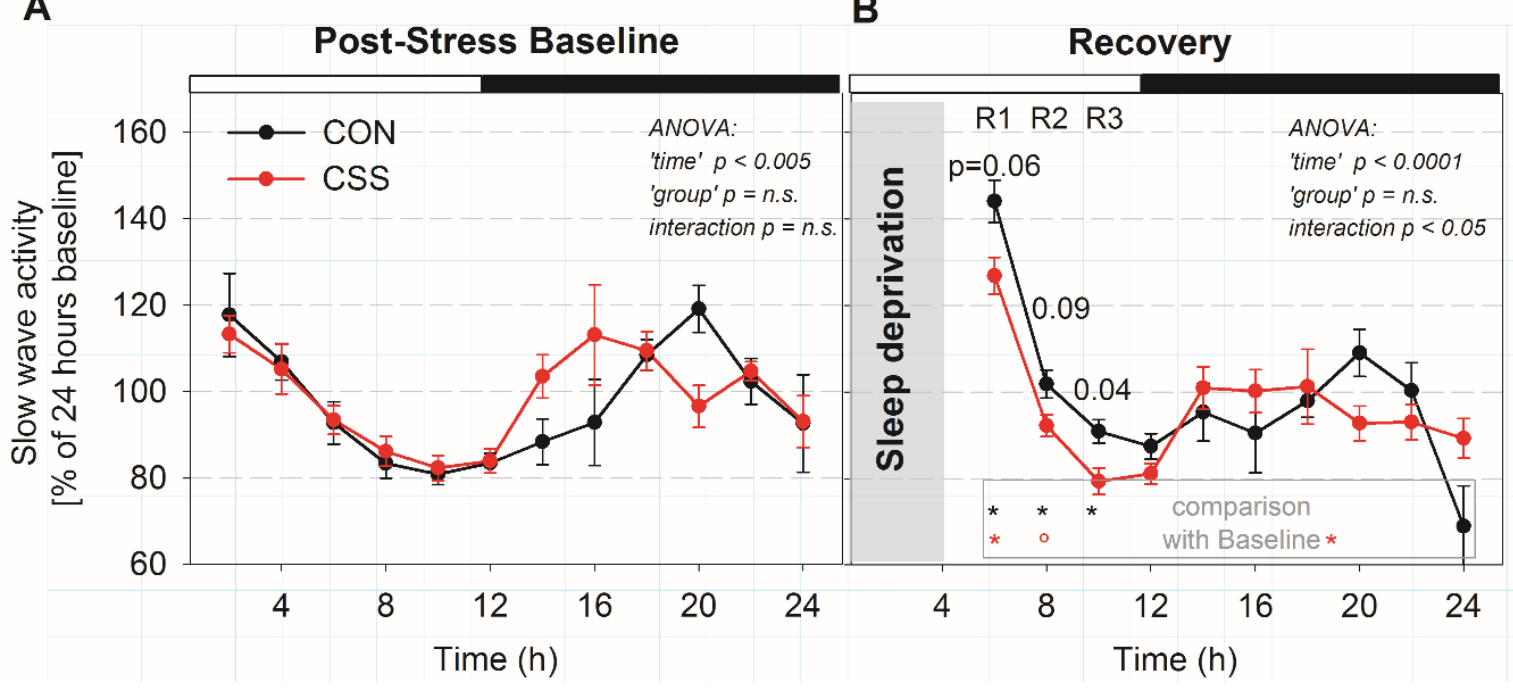

571

572

573

574

575 Figure 5:
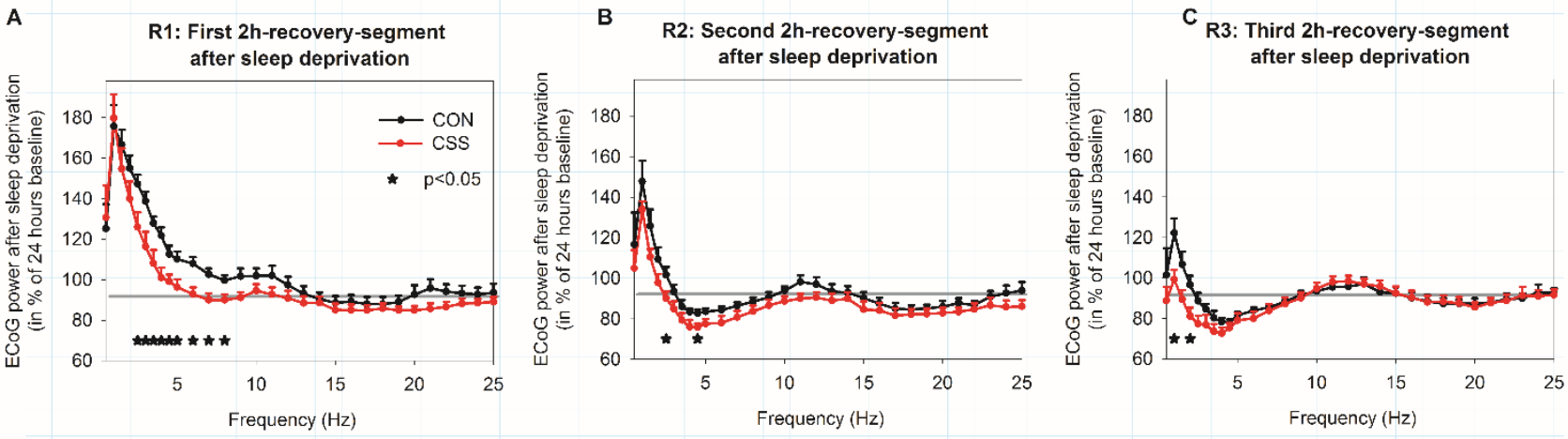